УДК $515.17+517.545$

\title{
Vector Bundle of Prym Differentials over Teichmüller Spaces of Surfaces with Punctures
}

\author{
Alexander V. Chueshev \\ Victor V. Chueshev* \\ Institute of Fundamental Sciences \\ Kemerovo State University \\ Krasnaya, 6, Kemerovo, 650043
}

Russia

Received 12.10.2018, received in revised form 18.01.2019, accepted 04.03.2019

In this paper we study multiplicative meromorphic functions and differentials on Riemann surfaces of finite type. We prove an analog of P. Appell's formula on decomposition of multiplicative functions with poles of arbitrary multiplicity into a sum of elementary Prym integrals. We construct explicit bases for some important quotient spaces and prove a theorem on a fiber isomorphism of vector bundles and $n$ !-sheeted mappings over Teichmüller spaces. This theorem gives an important relation between spaces of Prym differentials (Abelian differentials) on a compact Riemann surfaces and on a Riemann surfaces of finite type.

Keywords: Teichmüller spaces for Riemann surfaces of finite type, Prym differentials, vector bundles, group of characters, Jacobi manifolds.

DOI: 10.17516/1997-1397-2019-12-3-263-275.

\section{Introduction}

In the paper we study multiplicative meromorphic functions and differentials on Riemann surfaces of type $(g, n)$. Recently the interest in this subject has increased in relation with applications in theoretical physics, in particular, in description of vortex-like patterns in ferromagnetics [1].

Theory of functions on compact Riemann surfaces differs significantly from that on Riemann surfaces of finite type even for the class of single-valued meromorphic functions and Abelian differentials, since some of basic spaces of functions and differentials on Riemann surfaces $F^{\prime}$ of type $(g, n), g \geqslant 1, n>0$ are infinite-dimensional.

In this paper we continue constructing the general theory of functions on Riemann surfaces of type $(g, n)$ for multiplicative meromorphic function and differentials. We prove an analog of P. Appell's formula about the expansion of a multiplicative function with poles of arbitrary multiplicity into a sum of elementary Prym integrals. Also we construct explicit bases for some important quotient spaces and prove a theorem about fiber isomorphism of vector bundles and $n$ !-sheeted mappings over Teichmüller spaces. This theorem gives an important relation between spaces of Prym differentials (abelian differentials) on compact Riemann surfaces and Riemann surfaces of finite type.

*vvchueshev@ngs.ru

(C) Siberian Federal University. All rights reserved 


\section{Preliminaries}

Let $F$ be a smooth compact oriented surface of genus $g \geqslant 2$, with the marking $\left\{a_{k}, b_{k}\right\}_{k=1}^{g}$, i.e. an ordered collection of standard generators of $\pi_{1}(F)$, and $F_{0}$ be a compact Riemann surface with the fixed complex-analytic structure on $F$. Fix different points $P_{1}, \ldots, P_{n} \in F$. We assign type $(g, n)$ to a surface $F^{\prime}=F \backslash\left\{P_{1}, \ldots, P_{n}\right\}$. By $\Gamma^{\prime}$ we denote the Fuchsian group of genus 1 acting invariantly in the disk $U=\{z \in \mathbb{C}:|z|<1\}$ and uniformizing the surface $F_{0}^{\prime}$. Thus, $F_{0}^{\prime}=U / \Gamma^{\prime}$, where $\Gamma^{\prime}$ has the representation $[2,3]$

$$
\Gamma^{\prime}=\left\langle A_{1}, \ldots, A_{g}, B_{1}, \ldots, B_{g}, C_{1}, \ldots, C_{n}: \prod_{j=1}^{g} A_{j} B_{j} A_{j}^{-1} B_{j}^{-1} C_{1} \ldots C_{n}=I\right\rangle .
$$

Any other complex analytic structure on $F^{\prime}$ is given by a Beltrami differential $\mu$ on $F_{0}^{\prime}$, i. e. by an expression of the form $\mu(z) d \bar{z} / d z$, invariant with respect to the choice of the local parameter on $F_{0}^{\prime}$, where $\mu(z)$ is a complex-valued function on $F_{0}^{\prime}$ and $\|\mu\|_{L_{\infty}\left(F_{0}^{\prime}\right)}<1$. We denote this structure on $F^{\prime}$ by $F_{\mu}^{\prime}$.

Let $M\left(F^{\prime}\right)$ be the set of all complex analytic structures on $F^{\prime}$ with the topology of $C^{\infty}$ convergence on $F_{0}^{\prime}$, Diff $f^{+}\left(F^{\prime}\right)$ be the group of all orientation preserving smooth diffeomorphisms of $F^{\prime}$ onto itself, which leave all punctures fixed, and $D$ if $f_{0}\left(F^{\prime}\right)$ be the normal subgroup of $\operatorname{Diff} f^{+}\left(F^{\prime}\right)$ of diffeomorphisms homotopic to the identity diffeomorphism on $F_{0}^{\prime}$. The group $\operatorname{Diff} f^{+}\left(F^{\prime}\right)$ acts on $M\left(F^{\prime}\right)$ by $\mu \rightarrow f^{*} \mu$, where $f \in D i f f^{+}\left(F^{\prime}\right), \mu \in M\left(F^{\prime}\right)$. Then the Teichmüller space $\mathbb{T}_{g, n}\left(F_{0}^{\prime}\right)$ is the quotient space $M\left(F^{\prime}\right) / D$ if $f_{0}\left(F^{\prime}\right)[2]$.

Since the mapping $U \rightarrow F_{0}^{\prime}=U / \Gamma^{\prime}$ is a local diffeomorphism, any Beltrami differential $\mu$ on $F_{0}^{\prime}$ lifts to a Beltrami $\Gamma^{\prime}$-differential $\mu$ on $U$, i. e. $\mu \in L_{\infty}(U),\|\mu\|_{\infty}=\operatorname{esssup}_{z \in U}|\mu(z)|<1$, and $\mu(T(z)) \overline{T^{\prime}(z)} / T^{\prime}(z)=\mu(z), z \in U, T \in \Gamma^{\prime}$, see [2].

If the $\Gamma^{\prime}$-differential $\mu$ on $U$ is continued on $\overline{\mathbb{C}} \backslash U$, setting $\mu=0$, then there is a single quasiconformal homeomorphism $w^{\mu}: \overline{\mathbb{C}} \rightarrow \overline{\mathbb{C}}$ with fixed points $+1,-1, i$, which is a solution of the Beltrami equation $w_{\bar{z}}=\mu(z) w_{z}$. The map $T \rightarrow T_{\mu}=w^{\mu} T\left(w^{\mu}\right)^{-1}$ defines an isomorphism of the group $\Gamma^{\prime}$ onto the quasi-Fuchsian group $\Gamma_{\mu}^{\prime}=w^{\mu} \Gamma^{\prime}\left(w^{\mu}\right)^{-1}$.

In the work $\left[2\right.$, p. 99] there were constructed abelian differentials $\zeta_{1}[\mu], \ldots, \zeta_{g}[\mu]$ on $F_{[\mu]}$, that form a canonical base dual to a canonical homotopy base $\left\{a_{k}^{\mu}, b_{k}^{\mu}\right\}_{k=1}^{g}$ on $F_{\mu}$, which depends holomorphically on moduli $[\mu]$ for a class of conformal equivalency of a marked Riemann surface $F_{\mu}$. Further on, for brevity we shall write simply $F_{\mu}$ for the class of equivalence $F_{[\mu]}$. Here we assume that the class $[\mu]$ has Bers coordinates $h_{1}, h_{2}, \ldots, h_{3 g-3}$ when embedding the Teichmüller space $\mathbb{T}_{g}\left(F_{0}\right)$ of compact Riemann surfaces into $\mathbb{C}^{3 g-3}$. Moreover, the matrix of $b$-periods $\Omega(\mu)=\left(\pi_{j k}[\mu]\right)_{j, k=1}^{g}$ on $F_{\mu}$ consists of complex numbers $\pi_{j k}[\mu]=\int_{\xi}^{B_{k}^{\mu}(\xi)} \zeta_{j}([\mu], w) d w, \xi \in w^{\mu}(U)$, and depends holomorphically on $[\mu]$.

For any fixed $[\mu] \in \mathbb{T}_{g}$ and $\xi_{0} \in w^{\mu}(U)$ define the classical Jacobi mapping $\varphi: w^{\mu}(U) \rightarrow \mathbb{C}^{g}$ by the rule: $\varphi_{j}(\xi)=\int_{\xi_{0}}^{\xi} \zeta_{j}([\mu], w) d w, j=1, \ldots, g$. The quotient space $J(F)=\mathbb{C}^{g} / L(F)$ is called the marked Jacobi manifold for $F=F_{0}$, where $L(F)$ is a lattice over $\mathbb{Z}$, generated by the columns $e^{(1)}, \ldots, e^{(g)}, \pi^{(1)}, \ldots, \pi^{(g)}$ of the matrix $\left(I_{g}, \Omega\right)$, where $I_{g}$ is an identity matrix of order $g$. The universal Jacobi manifold of order $g$ is a fibered space over $\mathbb{T}_{g}$, with a fiber over $[\mu] \in \mathbb{T}_{g}$ being a marked Jacobi manifold $J\left(F_{\mu}\right)$ for a marked Riemann surface $F_{\mu}$ [4].

A character $\rho$ for $F_{\mu}^{\prime}$ is any homomorphic $\rho:\left(\pi_{1}\left(F_{\mu}^{\prime}\right), \cdot\right) \rightarrow\left(\mathbb{C}^{*}, \cdot\right), \mathbb{C}^{*}=\mathbb{C} \backslash\{0\}$. Further on we shall assume that $\rho\left(\gamma_{j}^{\mu}\right)=1$, where $\gamma_{j}^{\mu}$ is a simple loop around only one puncture $P_{j}$ on $F_{\mu}^{\prime}$, 
$j=1, \ldots, n$.

Definition 1. A multiplicative function $f$ on $F_{\mu}^{\prime}$ for the character $\rho$ is a mermorphic function $f$ on $w^{\mu}(U)$ such that $f(T z)=\rho(T) f(z), z \in w^{\mu}(U), T \in \Gamma_{\mu}^{\prime}$.

Definition 2. A Prym q-differential with respect to a Fuchsian group $\Gamma^{\prime}$ for $\rho$, or a $(\rho, q)$ differential, is a differential $\omega(z) d z^{q}$ such that $\omega(T z)\left(T^{\prime} z\right)^{q}=\rho(T) \omega(z), z \in U, T \in \Gamma^{\prime}, \rho$ : $\Gamma^{\prime} \rightarrow \mathbb{C}^{*}$.

If a multipicative function $f_{0}$ on $F_{\mu}$ for $\rho$ does not have zeroes or poles, then the character $\rho$ is called non-essential and $f_{0}$ is called a unit. The characters which are not non-essential are called essential on $\pi_{1}\left(F_{\mu}\right)$. The set $L_{g}$ of non-essential characters form a subgroup in the group $\operatorname{Hom}\left(\Gamma_{\mu}, \mathbb{C}^{*}\right)$ of all characters on $\Gamma_{\mu}$. A divisor on $F_{\mu}$ is a formal product $D=P_{1}^{n_{1}} \ldots P_{k}^{n_{k}}$, $P_{j} \in F_{\mu}, n_{j} \in \mathbb{Z}, j=1, \ldots, k$.

Theorem (Abel's theorem for characters, $[3,5]$ ). Let $D$ be a divisor on a marked variable compact Riemann surface $\left[F_{\mu},\left\{a_{1}^{\mu}, \ldots, a_{g}^{\mu}, b_{1}^{\mu}, \ldots, b_{g}^{\mu}\right\}\right]$ of genus $g \geqslant 1$, and $\rho$ be a character on $\pi_{1}\left(F_{\mu}\right)$. Then $D$ is a divisor of a multiplicative function $f$ on $F_{\mu}$ for $\rho$ if and only if deg $D=0$ and

$$
\varphi(D)=\frac{1}{2 \pi i} \sum_{j=1}^{g} \log \rho\left(b_{j}^{\mu}\right) e^{(j)}-\frac{1}{2 \pi i} \sum_{j=1}^{g} \log \rho\left(a_{j}^{\mu}\right) \pi^{(j)}[\mu](\equiv \psi(\rho,[\mu])),
$$

where $\varphi[\mu]: F_{\mu} \rightarrow J\left(F_{\mu}\right)$ is the Jacobi mapping.

The class $M_{1}(\rho)$ consists of those Prym differentials for $\rho$ on $F_{\mu}^{\prime}$, which have finitely many poles on $F_{\mu}^{\prime}$ and admit meromorphic continuation to $F_{\mu}$.

In [6] it was proved that for any essential character $\rho$, a point $Q_{1} \in F_{\mu}$, and natural $q \geqslant 1$ or a non-essential character $\rho$, a point $Q_{1} \in F_{\mu}$, and natural $q>1$ there exists an elementary $(\rho, q)$-differential $\tau_{\rho, q ; Q_{1}}$ of the third kind with a unique simple pole $Q_{1}[\mu]$ on $F_{\mu}$. For any nonessential character $\rho$, a point $Q_{1} \in F_{\mu}$ if $q=1$ there is no elementary $(\rho, 1)$-differential $\tau_{\rho ; Q_{1}}$. Also it is proved there that on a variable surface $F_{\mu}$ of genus $g \geqslant 2$ for any natural $q \geqslant 1$ there exists an elementary $(\rho, q)$-differential $\tau_{\rho, q ; Q_{1} Q_{2}}$ of the third kind with simple poles $Q_{1}, Q_{2} \in F_{\mu}$, and $\tau_{\rho, q ; Q_{1}}^{(m)}=\left(\frac{1}{z^{m}}+O(1)\right) d z^{q}, z\left(Q_{1}\right)=0$, of the second kind with the pole $Q_{1}[\mu]$ of order $m \geqslant 2$. These differentials depend locally holomorphically on $[\mu]$ and $\rho$.

Let $p: E \rightarrow B$ be a locally trivial holomorphic vector bundle of rank $m$, i.e. $E, B$ are complex analytic manifolds, the base $B$ is covered by a system of open simply-connected sets $\left\{U_{\alpha}\right\}$ such that there exists a system of holomorphic fiber coordinate homeomorphisms $\varphi_{\alpha}: U_{\alpha} \times \mathbb{C}^{m} \rightarrow$ $p^{-1}\left(U_{\alpha}\right)$ for all $\alpha$. On intersections $U_{\alpha} \cap U_{\beta} \neq \emptyset$ there are given $\varphi_{\beta \alpha}=\varphi_{\beta}^{-1} \varphi_{\alpha}:\left(U_{\alpha} \cap U_{\beta}\right) \times \mathbb{C}^{m} \rightarrow$ $\left(U_{\alpha} \cap U_{\beta}\right) \times \mathbb{C}^{m}$, holomorphic matrix transition functions, which satisfy on $\left(U_{\alpha} \cap U_{\beta} \cap U_{\gamma}\right) \times \mathbb{C}^{m}$ the relations $\varphi_{\alpha \gamma} \varphi_{\gamma \beta} \varphi_{\beta \alpha}=i d$, where $i d$ is the identity homeomorphism for all indices. The transition functions $\varphi_{\beta \alpha}(x, z)=\left(x, \widetilde{\varphi}_{\beta \alpha}(x) z\right)$ define holomorphic mappings $\widetilde{\varphi}_{\beta \alpha}: U_{\alpha} \cap U_{\beta} \rightarrow G L(m, \mathbb{C})$, where $x \in B, z \in \mathbb{C}^{m}$ и $\widetilde{\varphi}_{\alpha \gamma} \widetilde{\varphi}_{\gamma \beta} \widetilde{\varphi}_{\beta \alpha}=1$. These conditions on $B, \mathbb{C}^{m},\left\{U_{\alpha}\right\}$ and such $\varphi_{\beta \alpha}, \widetilde{\varphi}_{\beta \alpha}$ are sufficient to define a locally trivial holomorphic vector bundle $E$ of rank $m$ over $B$ [7].

Any holomorphic section $s: B \rightarrow E$, i.e. $p s(x)=x, x \in B$, may locally be described as $\varphi_{\alpha}^{-1} s: U_{\alpha} \rightarrow U_{\alpha} \times \mathbb{C}^{m}$, which define holomorphic vector-valued functions $s_{\alpha}: U_{\alpha} \rightarrow \mathbb{C}^{m}$ by the formula $\left(\varphi_{\alpha}^{-1} s\right)(x)=\left(x, s_{\alpha}(x)\right), x \in U_{\alpha}$. On intersections $U_{\alpha} \cap U_{\beta} \neq \emptyset$ these functions satisfy the compatibility conditions $s_{\beta}(x)=\widetilde{\varphi}_{\beta \alpha}\left(s_{\alpha}(x)\right)$.

Conversely, given a set of holomorphic vector-valued functions $s_{\alpha}: U_{\alpha} \rightarrow \mathbb{C}^{m}$ with the compatibility conditions satisified, then the formula $s(x)=\varphi_{\alpha}\left(x, s_{\alpha}(x)\right)$ uniquely, i.e. independently of the choice of the covering $\left\{U_{\alpha}\right\}$, defines a holomorphic section $s: B \rightarrow E$. 
If $E$ is a locally trivial holomorphic vector bundle of rank $m$ over $B$, then there exists a base of locally holomorphic sections for $\left\{U_{\alpha}\right\}$ given by $s_{k \alpha}=\varphi_{\alpha}\left(x, e_{k}\right), k=1, \ldots, m, x \in U_{\alpha}$, where $e_{1}, \ldots, e_{m}$ is the standard base in $\mathbb{C}^{m}$.

Conversely, given a base of locally holomorphic sections $s_{k \alpha}, k=1, \ldots, m, x \in U_{\alpha}$ of $E$, the coordinate homeomorphisms can be defined by $\varphi_{\alpha}(x, z)=\sum_{j=1}^{m} z_{j} s_{j \alpha}$, where $z=\sum_{j=1}^{m} z_{j} e_{j}$, which are holomorphic in $x \in B$ and $z \in \mathbb{C}^{m}$. Besides, from $\left(s_{1 \alpha}(x), \ldots, s_{m \alpha}(x)\right)^{t}=\widetilde{\varphi}_{\alpha \beta}(x)\left(s_{1 \beta}(x), \ldots\right.$ $\left.\ldots, s_{m \beta}(x)\right)^{t}$ it follows that the transition functions $\widetilde{\varphi}_{\alpha \beta}(x)$ are holomorphic on intersections $U_{\alpha} \cap U_{\beta} \neq \emptyset$. In this manner $(E, p, B)$ is endowed with the structure of a holomorphic vector bundle of rank $m$ over $B$.

\section{An analog of Appel's decomposition formula for a multiplicative function on a variable Riemann surface of finite type}

Denote by $T_{\rho ; Q}^{(1)}=-\int_{Q_{0}}^{P} \tau_{\rho ; Q}^{(2)}$ an elementary Prym integral of second kind on $F_{\mu}$ for an essential character $\rho$ with only simple pole at $Q$ and with residue +1 in $Q$ that depends holomorphically on $[\mu]$ and $\rho$, where $\tau_{\rho ; Q}^{(2)}$ has zero residue at $Q[5,6,8]$.

Let $f$ be a function on $F_{\mu}{ }^{\prime}$ of the class $M_{1}$ for an essential character $\rho$ with $s$ simple poles $P_{n+1}, P_{n+2}, \ldots, P_{n+s}$ and residues $c_{n+1}, \ldots, c_{n+s}$ at these poles respectively for some its branch. Consider an analytic continuation of this function $f$ (denoting it by the same symbol) from $F_{\mu}{ }^{\prime}$ to $F_{\mu}$. Consider the expression $f_{1}=f-c_{n+1} T_{\rho ; P_{n+1}}^{(1)}-\cdots-c_{n+s} T_{\rho ; P_{n+s}}^{(1)}-\sum_{j=1}^{g-1} \widetilde{c}_{j} \int_{Q_{0}}^{P} \tilde{\zeta}_{j}$, where $\widetilde{c}_{j} \in \mathbb{C}$, $j=1, \ldots, g-1$, and $\tilde{\zeta}_{1}, \ldots, \tilde{\zeta}_{g-1}$ is the base of Prym differentials of the first kind for an essential character $\rho$ on $F_{\mu}$ depending holomorphically on $[\mu]$ end $\rho$ [2]. Then $f_{1}$ is a meromorphic singlevalued branch of the Prym integral with an essential character $\rho$ on the fundamental polygon $\triangle_{\mu}$, where the surface $F_{\mu}$ is uniformized [3], with the divisor $\left(f_{1}\right) \geqslant \frac{1}{P_{1}^{q_{1}} \ldots P_{n}^{q_{n}}}, q_{j} \geqslant 0, j=1, \ldots, n$, on $F_{\mu}$. Here we assume $P_{1}, P_{2}, \ldots, P_{n+s} \in$ Int $\triangle_{\mu}$. Besides, the Prym integral $f_{1}$ for $\rho$ has a branch whose principal parts of Laurent series coincide with principal parts of Laurent series at $P_{j}, j=1, \ldots, n$, for $f$ and zero $a_{m}$-periods, $m=1, \ldots, g-1$, on $F_{\mu}$ or on $\triangle_{\mu}[2]$. Therefore $f=\sum_{j=1}^{s} c_{n+j} T_{\rho ; P_{n+j}}^{(1)}+\sum_{j=1}^{g-1} \widetilde{c}_{j} \int_{Q_{0}}^{P} \tilde{\zeta}_{j}+f_{1}$.

If $P_{l}$ is a pole of order $q_{l}, q_{l} \geqslant 2$, then in the formula above one should instead of $c_{l} T_{\rho ; P_{l}}^{(1)}$, $l=n+1, \ldots, n+s$, (for simple poles), and also for poles $P_{l}, l=1, \ldots, n$, of the branch of $f_{1}$ write sums of the form

$$
A_{l, 1} T_{\rho ; P_{l}}^{(1)}+A_{l, 2} \frac{\partial T_{\rho ; P_{l}}^{(1)}}{\partial P_{l}}+\frac{A_{l, 3}}{2} \frac{\partial^{2} T_{\rho ; P_{l}}^{(1)}}{\partial P_{l}^{2}}+\cdots+\frac{A_{l, q_{l}}}{\left(q_{l}-1\right) !} \frac{\partial^{q_{l}-1} T_{\rho ; P_{l}}^{(1)}}{\partial P_{l}^{q_{l}-1}},
$$

where $A_{l, j}$ are coefficients of the principal part of the Laurent series for some branch of $f$ at $P_{l}, j=1, \ldots, q_{l}\left(P_{l}\right), l=n+1, \ldots, n+s$, and for a branch of $f_{1}$ at $P_{1}, P_{2}, \ldots, P_{n}$. Indeed, in a neighborhood of $P_{l}$ we have expansions $T_{\rho ; P_{l}}^{(1)}=\frac{1}{z-z\left(P_{l}\right)}+O(1) ;\left(T_{\rho ; P_{l}}^{(1)}\right)_{a_{l}}^{\prime}=\frac{1}{\left(z-a_{l}\right)^{2}}+O(1)$, $z\left(P_{l}\right)=a_{l} ; \ldots ;\left(T_{\rho ; P_{l}}^{(1)}\right)_{a_{l}}^{(m)}=\frac{m !}{\left(z-a_{l}\right)^{m+1}}+O(1), 1 \leqslant m \leqslant q_{l}\left(P_{l}\right)-1$, where $q_{l}\left(P_{l}\right)$ is the order 
of the pole at $P_{l}$ for branches $f$ and $f_{1}, l=1, \ldots, s+n$. From that follows the theorem.

Theorem 1. Let $f$ be a branch of a function of class $M_{1}$ for an essential character $\rho$ on a variable Riemann surface $F_{\mu}^{\prime}$ of type $(g, n), g \geqslant 2, n>0$, with pairwise distinct poles at $P_{n+1}, \ldots, P_{n+s}$ of multiplicities $q_{n+1}, \ldots, q_{n+s}$ with given principal parts:

$$
\frac{A_{j, q_{j}}}{\left(z-z\left(P_{j}\right)\right)^{q_{j}}}+\ldots+\frac{A_{j 1}}{\left(z-z\left(P_{j}\right)\right)}, \quad j=n+1, \ldots, n+s .
$$

Then for an analytic continuation of $f$ we have $(f) \geqslant \frac{1}{P_{1}^{q_{1}} \ldots P_{n+s}^{q_{n+s}}}, q_{j} \geqslant 0, j=1, \ldots, n$, on $F_{\mu}$ and

$$
f=\sum_{j=1}^{n+s} \sum_{m=1}^{q_{j}}\left[\frac{A_{j, m}}{(m-1) !} \frac{\partial^{m-1} T_{\rho ; P_{j}}^{(1)}}{\partial P_{j}^{m-1}}\right]+\sum_{j=1}^{g-1} \widetilde{c}_{j} \int_{Q_{0}}^{P} \tilde{\zeta}_{j},
$$

where $f=\frac{A_{j, q_{j}}}{\left(z-z\left(P_{j}\right)\right)^{q_{j}}}+\ldots+\frac{A_{j, 2}}{\left(z-z\left(P_{j}\right)\right)^{2}}+\frac{A_{j, 1}}{z-z\left(P_{j}\right)}+O(1)$ for some branch in a neighborhood of $P_{j}, j=1, \ldots, n+s$, на $F_{\mu}$, and all summands depend holomorphically on $[\mu]$ and $\rho$.

Let now $\rho$ be a non-essential character. The proof of the previous expansion formula for an essential character does not work since in this case there is no Prym integral of the second kind with only simple pole on $F_{\mu}$. Therefore we need a Prym differential $\tau_{\rho ; Q_{1}^{2} Q_{2}^{2}}$ of second kind for a non-essential character $\rho$ with two poles of second order at two distinct points $Q_{1}$ and $Q_{2}$ on $\triangle_{\mu}$ with zero residues at $Q_{1}$ and $Q_{2}[5,6]$. In this case one should use as basic elements of expansion the Prym integrals $T_{\rho ; Q_{1} Q_{2}}=-\int_{Q_{0}}^{P} \tau_{\rho ; Q_{1}^{2} Q_{2}^{2}}$ of second kind with two simple poles $Q_{1}$ and $Q_{2}$.

Consider one more Prym differential $\tau_{\rho ; Q_{1} Q_{2}}=f_{0} \tau_{Q_{1} Q_{2}}$ of the third kind on $F_{\mu}$, where $f_{0}$ is a unit for $\rho$ on $F_{\mu}$ and $\tau_{Q_{1} Q_{2}}$ is the normalized (i.e all $a$-periods vanish) abelian differential with simple poles $Q_{1}$ and $Q_{2}$ on $F_{\mu}$ and residues +1 and -1 at these points, respectively, which depend holomorphically on $[\mu]$ and $\rho[5,6]$. It is known that $\tau_{Q_{1} Q_{2}}=d \Pi_{Q_{1} Q_{2}}$ and the abelian integral $\Pi_{Q_{1} Q_{2}}$ can be expressed implicitly via the Riemann theta-function for the surface $F_{\mu}$. It equals to a sum of two functions, one of which depends only on $Q_{1}$, and another only on $Q_{2}$ [5, p. 117]. Therefore the derivative $\frac{\partial \Pi_{Q_{1} Q_{2}}}{\partial z_{1}}$ does not depend on $Q_{2}$, where $z_{1}=z\left(Q_{1}\right)$.

The Prym differential $\tau_{\rho ; Q_{1}}^{(2)}$ admits the expansion $\left(\frac{1}{\left(z-z_{1}\right)^{2}}+\frac{c_{-1}^{(1)}}{z-z_{1}}+O(1)\right) d z$ in a neighborhood of $Q_{1}, z\left(Q_{1}\right)=z_{1}$, where $c_{-1}^{(1)}=\sum_{j=1}^{g} \log \rho\left(a_{j}\right) \varphi_{j}^{\prime}\left(Q_{1}\right)[5,6]$. To prove this we consider the abelian differential $\frac{\tau_{\rho, Q}^{(2)}}{f_{0}}$, where $f_{0}$ is a multiplicative unit for $\rho$. Its complete sum of residues is zero. In a neighborhood of $Q, z(Q)=z_{0}$ we have the Laurent expansions

$$
\begin{gathered}
\tau_{\rho, Q}^{(2)}=\left(\frac{1}{\left(z-z_{0}\right)^{2}}+\frac{c_{-1}}{z-z_{0}}+c_{0}+\ldots\right) d z \\
\frac{1}{f_{0}(z)}=\exp \left(-\sum_{j=1}^{g} \lambda_{j} \varphi_{j}(z)\right)=\frac{1}{f_{0}\left(z_{0}\right)}-\frac{\left(z-z_{0}\right)}{f_{0}\left(z_{0}\right)}\left(\lambda_{1} \varphi_{1}^{\prime}\left(z_{0}\right)+\ldots+\lambda_{g} \varphi_{g}^{\prime}\left(z_{0}\right)\right)+\ldots
\end{gathered}
$$

where $\lambda_{j}=\log \rho\left(a_{j}\right), j=1, \ldots, g$. From that we get

$$
0=\operatorname{res}_{z_{0}} \frac{\tau_{\rho, Q}^{(2)}}{f_{0}}=\frac{c_{-1}}{f_{0}\left(z_{0}\right)}-\frac{\lambda_{1} \varphi_{1}^{\prime}\left(z_{0}\right)+\cdots+\lambda_{g} \varphi_{g}^{\prime}\left(z_{0}\right)}{f_{0}\left(z_{0}\right)},
$$


since $Q$ is the only pole of the abelian differential. Therefore $c_{-1}=\sum_{j=1}^{g} \lambda_{j} \varphi_{j}^{\prime}\left(z_{0}\right)$ and $c_{-1}=0$ in a finite number of points $Q$ on $\triangle_{\mu}$. Indeed, $d f_{0}=\exp \left(\sum_{j=1}^{g} \lambda_{j} \varphi_{j}(P)\right) \sum_{j=1}^{g} \lambda_{j} \varphi_{j}^{\prime} d z(P)$, and the equivalency $\sum_{j=1}^{g} \lambda_{j} \varphi_{j}^{\prime}(Q)=0 \Leftrightarrow d f_{0}(Q)=0$ holds. Thus, for a non-essential character $\rho$ there is no Prym differential of the second kind with only pole of the second order at an arbitrary point $Q$ and principal part $\frac{1}{\left(z-z_{0}\right)^{2}}$, since the condition $\lambda_{1} \varphi_{1}^{\prime}\left(z_{0}\right)+\cdots+\lambda_{g} \varphi_{g}^{\prime}\left(z_{0}\right)=0$ holds only for a finite number of points $Q$ on $\triangle_{\mu}$, i.e. at points $Q$ that are zeroes of the differential $d f_{0}$.

The Prym differential $\tau_{\rho ; Q_{2}}^{(2)}$ also has an expansion $\left(\frac{1}{\left(z-z_{2}\right)^{2}}+\frac{c_{-1}^{(2)}}{z-z_{2}}+O(1)\right) d z$ in a neighborhood of $Q_{2}$ on $F_{\mu}$, where $c_{-1}^{(2)}=\sum_{j=1}^{g} \log \rho\left(a_{j}\right) \varphi_{j}^{\prime}\left(Q_{2}\right)$.

A Prym differentials with two poles of the second order and zero residues at these points may be given in the form

$$
\tau_{\rho ; Q_{1}^{2} Q_{2}^{2}}=c_{-1}^{(2)} f_{0}\left(Q_{1}\right) \tau_{\rho ; Q_{1}}^{(2)}-c_{-1}^{(1)} f_{0}\left(Q_{2}\right) \tau_{\rho ; Q_{2}}^{(2)}-c_{-1}^{(1)} c_{-1}^{(2)} \tau_{\rho ; Q_{1} Q_{2}} .
$$

Note that the principal part for $\tau_{\rho, Q_{1} Q_{2}}$ at $Q_{1}$ has the form $\frac{f_{0}\left(Q_{1}\right)}{z-z_{1}}$, and at $Q_{2}$ it is $-\frac{f_{0}\left(Q_{2}\right)}{z-z_{2}}$. It follows that the differential constructed above $\tau_{\rho ; Q_{1}^{2} Q_{2}^{2}}$ has poles of the second order at ${ }_{2}$ and $Q_{2}$, and zero residues at these points. Indeed, in a neighborhood of $Q_{1}$ its principal part has the form $c_{-1}^{(2)} f_{0}\left(Q_{1}\right)\left[\frac{1}{\left(z-z_{1}\right)^{2}}+\frac{c_{-1}^{(1)}}{z-z_{1}}\right]-c_{-1}^{(1)} c_{-1}^{(2)} \frac{f_{0}\left(Q_{1}\right)}{z-z_{1}}=\frac{c_{-1}^{(2)} f_{0}\left(Q_{1}\right)}{\left(z-z_{1}\right)^{2}}$; analogously at $Q_{2}$ : $\left(-c_{-1}^{(1)} f_{0}\left(Q_{2}\right)\left[\frac{1}{\left(z-z_{2}\right)^{2}}+\frac{c_{-1}^{(2)}}{z-z_{2}}\right]\right)+c_{-1}^{(1)} c_{-1}^{(2)} \frac{f_{0}\left(Q_{2}\right)}{z-z_{2}}=-\frac{c_{-1}^{(1)} f_{0}\left(Q_{2}\right)}{\left(z-z_{2}\right)^{2}}$. The constructed differential $\tau_{\rho ; Q_{1}^{2} Q_{2}^{2}}$ depend holomorphically on $[\mu]$ and $\rho$.

Theorem 2. Let $f$ be a branch of a function of class $M_{1}$ for a non-essential character $\rho$ on a variable Riemann surface $F_{\mu}^{\prime}$ of type $(g, n), g \geqslant 2, n>0$, with pairwise distinct poles at $P_{n+1}, \ldots, P_{n+s}$ of multiplicities $q_{n+1}, \ldots, q_{n+s}$ with given principal parts (1). Assume that for an analytic continuation of $f$ to $F_{\mu}$ the conditions $(f) \geqslant \frac{1}{P_{1}^{q_{1}} \ldots P_{n+s}^{q_{n+s}}}, q_{j} \geqslant 0, j=1, \ldots, n$, and $\sum_{j=1}^{g} \log \rho\left(a_{j}\right) \varphi_{j}^{\prime}\left(P_{n+s}\right) \neq 0$ are fulfilled. Then

$$
\begin{aligned}
f(P) & =\sum_{j=1}^{g} c_{j} \int_{Q_{0}}^{P} f_{0} \zeta_{j}+\sum_{r=1}^{n+s-1} \frac{A_{r 1} T_{\rho ; P_{r} P_{n+s}}}{d_{n+s} f_{0}\left(P_{r}\right)}+\sum_{m=2}^{q_{1}} \frac{A_{1 m}}{(m-1) !} \frac{\partial^{m-1} T_{\rho ; P_{1} P_{n+1}}}{\partial P_{1}^{m-1}}+ \\
& +\sum_{j=2}^{n+s}\left[A_{j, 2} \frac{\partial T_{\rho ; P_{j} P_{1}}}{\partial P_{j}}+\frac{A_{j, 3}}{2 !} \frac{\partial^{2} T_{\rho ; P_{j} P_{1}}}{\partial P_{j}^{2}}+\ldots+\frac{A_{j, q_{j}}}{\left(q_{j}-1\right) !} \frac{\partial^{q_{j}-1} T_{\rho ; P_{j} P_{1}}}{\partial P_{j}^{q_{j}-1}}\right]+C
\end{aligned}
$$

where

$$
f=\frac{A_{j, q_{j}}}{\left(z-z\left(P_{j}\right)\right)^{q_{j}}}+\ldots+\frac{A_{j, 2}}{\left(z-z\left(P_{j}\right)\right)^{2}}+\frac{A_{j, 1}}{z-z\left(P_{j}\right)}+O(1)
$$

for some branch in a neighborhood of $P_{j}, j=1, \ldots, n+s$, on $F_{\mu} ; C=0$ for $\rho \neq 1 ; d_{k}=$ $=\sum_{m=1}^{g} \log \rho\left(a_{m}\right) \varphi_{m}^{\prime}\left(P_{k}\right), k=1, \ldots, n+s$, on $F_{\mu}$, and all summands depend holomorphically on $[\mu]$ and $\rho$. 
Proof. It is enough to check that principal parts of both parts of the formula coincide. For a neighborhood of $P_{r}, r=1, \ldots, n+s-1$, on $\triangle_{\mu}$ we have the Laurent expansion

$$
\sum_{r=1}^{n+s-1}\left(\frac{d_{n+s} f_{0}\left(P_{r}\right)}{z-P_{r}}-\frac{d_{r} f_{0}\left(P_{n+s}\right)}{z-P_{n+s}}\right) \frac{A_{r 1}}{d_{n+s} f_{0}\left(P_{r}\right)}=\frac{A_{r 1}}{z-P_{r}}+\ldots
$$

For a neighborhood of $P_{n+s}$ on $\triangle_{\mu}$ we have

$$
\sum_{r=1}^{n+s-1} \frac{-d_{r} f_{0}\left(P_{n+s}\right)}{z-P_{n+s}} \frac{A_{r 1}}{d_{n+s} f_{0}\left(P_{r}\right)}=\frac{1}{z-P_{n+s}} \frac{f_{0}\left(P_{n+s}\right)}{d_{n+s}} \sum_{r=1}^{n+s-1} \frac{-d_{r} A_{r 1}}{f_{0}\left(P_{r}\right)}+\ldots=\frac{A_{n+s, 1}}{z-P_{n+s}}+\ldots
$$

since $\sum_{r=1}^{n+s} \frac{-A_{r 1} d_{r}}{f_{0}\left(P_{r}\right)}=0, \frac{f_{0}\left(P_{n+s}\right)}{d_{n+s}} \sum_{r=1}^{n+s-1} \frac{-d_{r} A_{r 1}}{f_{0}\left(P_{r}\right)}=A_{n+s, 1}$, according to the complete sum of residues formula for an abelian differential $\frac{f}{f_{0}} d\left(\sum_{j=1}^{g} \log \rho\left(a_{j}\right) \varphi_{j}\right)$ of the third kind on $F_{\mu}$, which at $P_{j}$ has the residues $\frac{A_{j 1} d_{j}}{f_{0}\left(P_{j}\right)}, j=1, \ldots, n+s$. Thus, the coefficients at the power -1 in principal parts at $P_{1}, P_{2}, \ldots, P_{n+s}$, are the same.

The third sum shows that the coefficients coincide in principal parts at $P_{1}$ for powers starting from -2 . The fourth sum shows that the coefficients coincide in principal parts at $P_{2}, P_{3}, \ldots, P_{n+s}$ for powers starting from -2 .

If $\rho=1$ then all the summands in the formula become abelian integrals, which differ by a constant $C$. If $\rho \neq 1$ and $\rho$ is a non-essential character, then $C=0$, since a constant is neither a multiplicative function, nor a Prym integral for this character on $F_{\mu}$ of genus $g \geqslant 2$.

Remark. P. Appel [6, see p. 118] proved Theorem 2 for a fixed compact Riemann surface and simple poles with every simple element (summand) depending on additional $g-1$ poles. Our theorem is proved for a variable Riemann surface $F^{\prime}$ of finite type $(g, n), g \geqslant 2, n>0$, and poles of any order with any summand having either one or two poles. Moreover, if $\rho=1, n=0$ we recover the classical fact on decomposition of a single-valued meromorphic function into a sum of abelian integrals on a compact Riemann surface.

Corollary. For any non-essential character $\rho$ on a variable compact Riemann surface $F_{\mu}$ of genus $g \geqslant 2$ at $Q_{1}$, which are zeroes of the differential $d f_{0}$, there exists a differential $\tau_{\rho, Q_{1}}^{(2)}$ of the second kind with only pole of the second order at $Q_{1}$ that depends holomorphically on $[\mu]$ and $\rho$, and having zero residue at $Q_{1}$.

\section{Vector bundles of Prym differentials over a Techmüller space of Riemann surfaces of finite type}

Denote by $\Omega_{\rho}^{q}\left(\frac{1}{Q_{1}^{\alpha_{1}} \cdots Q_{s}^{\alpha_{s}}} ; F_{\mu}\right)$ the vector space of $(\rho, q)$-differentials that are multiples of the divisor $\frac{1}{Q_{1}^{\alpha_{1}} \cdots Q_{s}^{\alpha_{s}}}$, where $\alpha_{j} \geqslant 1, \alpha_{j} \in \mathbb{N}, j=1, \ldots, s, s \geqslant 1, q \geqslant 1, q \in \mathbb{N}$, and by $\Omega_{\rho}^{q}\left(1 ; F_{\mu}\right)$ the vector subspace of holomorphic $(\rho, q)$-differentials on $F_{\mu}[3]$. Here the divisor $Q_{1} \ldots Q_{s}$ on $F_{\mu}$ is understood as a constant set of points on a surface $F$ of genus $g \geqslant 2$.

Let $\widetilde{E}$ be the principal $\operatorname{Hom}\left(\Gamma, \mathbb{C}^{*}\right)$-bundle over $\mathbb{T}_{g}\left(F_{0}\right)$ with the fiber $\operatorname{Hom}\left(\Gamma_{\mu}, \mathbb{C}^{*}\right)$ over $F_{[\mu]}$ from $\mathbb{T}_{g}\left(F_{0}\right)$. Here $F_{0}=U / \Gamma, \Gamma$ is a Fuchsian group uniformizing $F_{0}$ over the circle $U$, and $F_{[\mu]}=w^{\mu}(U) / \Gamma_{\mu}=\Delta_{\mu} / \Gamma_{\mu}, \Gamma_{\mu}$ be a quasi-Fuchsian group uniformizing the compact Riemann surface $F_{[\mu]}$ over $w^{\mu}(U)$. 
Lemma 1 ([5], pp. 105-106). A holomorphic principal Hom $\left(\Gamma, \mathbb{C}^{*}\right)$-bundle $\widetilde{E}$ is biholomorphic to the trivial bundle $\mathbb{T}_{g}\left(F_{0}\right) \times \operatorname{Hom}\left(\Gamma, \mathbb{C}^{*}\right)$ over $\mathbb{T}_{g}\left(F_{0}\right)$.

Proposition 1. The vector bundle $E=\cup \Omega_{\rho}^{q}\left(\frac{1}{Q_{1}^{\alpha_{1}} \cdots Q_{s}^{\alpha_{s}}} ; F_{\mu}\right) / \Omega_{\rho}^{q}\left(1 ; F_{\mu}\right)$ over $\mathbb{T}_{g} \times$ $\left(\operatorname{Hom}\left(\Gamma, \mathbb{C}^{*}\right) \backslash 1\right)$ for $q>1\left(\right.$ over $\mathbb{T}_{g} \times\left(\operatorname{Hom}\left(\Gamma, \mathbb{C}^{*}\right) \backslash L_{g}\right)$ when $\left.q=1\right)$ and $g \geqslant 2$ is a holomorphic vector bundle of rank $\alpha_{1}+\cdots+\alpha_{s}=d$, while the co-sets of $(\rho, q)$-differentials

$$
\tau_{\rho, q ; Q_{1}}^{(1)}, \ldots, \tau_{\rho, q ; Q_{1}}^{\left(\alpha_{1}\right)}, \ldots, \tau_{\rho, q ; Q_{s}}^{(1)}, \ldots, \tau_{\rho, q ; Q_{s}}^{\left(\alpha_{s}\right)},
$$

form a basis of locally holomorphic sections of this bundle.

Proof. With given conditions on $q$ for the character $\rho$ we have the equality $\operatorname{dim} \Omega_{\rho}^{q}\left(1 ; F_{\mu}\right)=$ $=(g-1)(2 q-1)$. By the Riemann-Roch theorem for $(\rho, q)$-differentials we find the dimension

$$
i_{\rho, q}\left(Q_{1}^{-\alpha_{1}} \ldots Q_{s}^{-\alpha_{s}} ; F_{\mu}\right)=(g-1)(2 q-1)+\alpha_{1}+\ldots+\alpha_{s}+r\left((f[\mu]) Z^{q-1} Q_{1}^{\alpha_{1}} \ldots Q_{s}^{\alpha_{s}}\right),
$$

where $f[\mu]$ is a function for $\rho, Z$ is the canonical class for abelian 1-differentials on $F_{\mu}$. Here $r\left((f[\mu]) Z^{q-1} Q_{1}^{\alpha_{1}} \cdots Q_{s}^{\alpha_{s}}\right)=0$, since $\operatorname{deg}\left((f[\mu]) Z^{q-1} Q_{1}^{\alpha_{1}} \cdots Q_{s}^{\alpha_{s}}\right) \geqslant \alpha_{1}>0$. Thus,

$$
\operatorname{dim} \Omega_{\rho}^{q}\left(\frac{1}{Q_{1}^{\alpha_{1}} \cdots Q_{s}^{\alpha_{s}}} ; F_{\mu}\right) / \Omega_{\rho}^{q}\left(1 ; F_{\mu}\right)=\alpha_{1}+\ldots+\alpha_{s}=d
$$

It follows from Theorems 2.1 and 2.2 of [6] that there exist differentials from the set (2) that depend locally holomorphically on $[\mu]$ and $\rho$.

Let us show that the set $(2)$ of equivalency classes of $(\rho, q)$-differentials that depend locally holomorphically on $[\mu]$ and $\rho$ is linearly independent over $\mathbb{C}$ for given characters $\rho$. Consider a linear combination of the form

$$
C_{1}^{(1)} \tau_{\rho, q ; Q_{1}}^{(1)}+\ldots+C_{1}^{\left(\alpha_{1}\right)} \tau_{\rho, q ; Q_{1}}^{\left(\alpha_{1}\right)}+\ldots+C_{s}^{(1)} \tau_{\rho, q ; Q_{s}}^{(1)}+\ldots+C_{s}^{\left(\alpha_{s}\right)} \tau_{\rho, q ; Q_{s}}^{\left(\alpha_{s}\right)}=\omega
$$

where $\omega$ is a holomorphic $(\rho, q)$-differential on $F_{\mu}$. Since the right hand side does not have singularities, all the coefficients are zeroes. All these differentials depend holomorphically on $[\mu], \rho$ and divisors $Q_{1} \ldots Q_{s}$, which are locally holomorphic (constant) sections of the bundle of integer divisors of degree $s$ over the Teichmüller space $\mathbb{T}_{g}$ of genus $g$ [4]. Therefore, this set gives the base of locally holomorphic sections of this bundle.

Lemma 2. For any divisor $P_{1}^{q_{1}} \cdot \ldots \cdot P_{n}^{q_{n}}, q_{j} \geqslant 0, j=1, \ldots, n, q>1$ and any $\rho$ (or $q=1$ and an essential character $\rho)$ on $F_{\mu}$ of genus $g \geqslant 2$, there exists a differential $\tilde{\omega} \in \Omega_{\rho}^{q}\left(\frac{1}{P_{1}^{q_{1}} \cdot \ldots \cdot P_{n}^{q_{n}}}, F_{\mu}\right)$ with the divisor $(\tilde{\omega})=\frac{R_{1}, \ldots, R_{N}}{P_{1}^{q_{1}} \cdot \ldots \cdot P_{n}^{q_{n}}}$, where $R_{j} \neq P_{l}, l=1, \ldots, n, j=1, \ldots, N, N=(2 g-2) q+$ $+q_{1}+\ldots+q_{n}$, and any given principal parts of Laurent series at $P_{j}, j=1, \ldots, n$, for its branches. This differential depends locally holomorphically on moduli $[\mu]$ of the surface $F_{\mu}$ and the character $\rho$.

Proof. If $q_{j}=0$ for all $j$, there exists a holomorphic $(\rho, q)$-differential $\tilde{\omega} \neq 0$ on $F_{\mu}$ for every $q \geqslant 1$ and $\rho$, since $i_{\rho, q}(1)=(2 q-1)(g-1) \geqslant 3$ if $q>1$ and $i_{\rho}(1) \geqslant g-1 \geqslant 1$ if $q=1$.

Fix $q_{1}, \ldots, q_{n}$ as possible order of poles at punctures $P_{1}, \ldots, P_{n}$ on $F_{\mu}$ respectively and assume that for at least one $j, q_{j} \geqslant 1$.

If $q=1$ and $q_{1}=1, q_{2}=0, \ldots, q_{n}=0$ for an essential character $\rho$ there exists a differential $\tilde{\omega} \neq 0$ such that $(\tilde{\omega}) \geqslant \frac{1}{P_{1}}[6]$. Further on, if $q=1$ we shall assume that $q_{1}+q_{2}+\cdots+q_{n} \geqslant 2$.

For any $(\rho, q)$-differential $\tilde{\omega}$ the degree of its divisor $\operatorname{deg}(\tilde{\omega})=(2 g-2) q$ on $F_{\mu}$. It follows that $N=(2 g-2) q+q_{1}+\ldots+q_{n}$. By Proposition 1.4.4 [5] and Abel's theorem 
there exists a differential $\tilde{\omega} \neq 0$ with the divisor $(\tilde{\omega})=\frac{R_{1}, \ldots, R_{N}}{P_{1}^{q_{1}} \cdot \ldots \cdot P_{n}^{q_{n}}}$ if and only if the equality $\varphi\left(R_{1} \ldots R_{N}\right)-\varphi\left(P_{1}^{q_{1}} \ldots P_{n}^{q_{n}}\right)=-2 K q+\psi(\rho)$ holds in the Jacobi manifold $J\left(F_{\mu}\right)$, where $K$ is the vector of Riemann constants. From this it follows that $\varphi\left(R_{1} \ldots R_{g}\right)=$ $=-2 K q+\varphi\left(P_{1}^{q_{1}} \ldots P_{n}^{q_{n}}\right)+\psi(\rho)-\varphi\left(R_{g+1} \ldots R_{N}\right)$. Thus, to determine the zeroes of the differential we have $N-g=(2 g-2) q-g+q_{1}+\ldots+q_{n} \geqslant g-1 \geqslant 1$ free parameters that can be chosen so that they depend locally holomorphically on moduli $[\mu]$. Solving the Jacobi inversion problem we find the divisor $R_{1} \ldots R_{g}$, which is the only holomorphic solution to the previous equation if the right hand side does not belong to $W_{g}^{1}[3,5]$. This can be done since $\operatorname{dim} W_{g}^{1} \leqslant g-2$, but $N-g>g-2$ under our hypothesis. Therefore the divisor of the differential $(\tilde{\omega})=\frac{R_{1} \ldots R_{N}}{P_{1}^{q_{1}} \ldots P_{n}^{q_{n}}}$ has exactly required singularities, if $R_{j} \neq P_{l}$ for all indices. In order to do this we choose points $R_{g+1}, \ldots, R_{N} \neq$ $P_{1}, \ldots, P_{n}$. We shall show that after a specific choice of the divisor $R_{g+1} \ldots R_{N}$ we can satisfy the condition $R_{j} \neq P_{l}$ for any $j$ and $l$. Assume the converse, if $R_{1}=P_{1}$ on $F_{\mu}$, then from the previous equality we get $\varphi\left(R_{2} \ldots R_{g}\right)=-2 K q+\varphi\left(P_{1}^{q_{1}-1} P_{2}^{q_{2}} \ldots P_{n}^{q_{n}}\right)+\psi(\rho)-\varphi\left(R_{g+1} \ldots R_{N}\right)$ or $\varphi\left(R_{2} \ldots R_{g} R_{g+1} \ldots R_{2 g-1}\right)=-2 K q+\varphi\left(P_{1}^{q_{1}-1} P_{2}^{q_{2}} \ldots P_{n}^{q_{n}}\right)+\psi(\rho)-\varphi\left(R_{2 g} \ldots R_{N}\right)$. Consider the integer divisor $D=R_{2} \ldots R_{g} R_{g+1} \ldots R_{2 g-1}$ of degree $2 g-2$. It has $g-1$ free points $R_{g+1}, \ldots, R_{2 g-1}$. By the free points theorem [3] we get the inequality $i(D) \geqslant 1$, and therefore $\varphi(D)=-2 K$. Then the previous inequality can be rewritten as

$$
-2 K(q-1)+\varphi\left(P_{1}^{q_{1}-1} \ldots P_{n}^{q_{n}}\right)+\psi(\rho)=\varphi\left(R_{2 g} \ldots R_{N}\right) .
$$

Note that $N-(2 g-1)=(2 g-2) q+q_{1}+\ldots+q_{n}-2 g+1 \geqslant 1$ in these conditions. Thus, we see that the sets defined by both sides of this equality in $J\left(F_{\mu}\right)$ has different dimensions. Therefore we can choose $R_{2 g}, \ldots, R_{N}$ on $F_{\mu}$ such that (3) does not hold. This is a contradiction.

It is known that under our conditions on $q$ and character $\rho$ there exist elementary $(\rho, q)$ differentials of the form $\tau_{\rho, q ; Q}^{(1)}$ and $\tau_{\rho, q ; Q}^{(m)}, m>1$ on $F_{\mu}[6]$. Therefore we can construct any principal parts for Laurent series of the differential $\tilde{\omega}$ at all points $P_{j}, j=1, \ldots, n$, on $F_{\mu}$.

Further on, we shall assume that the character $\rho^{\prime}$ on $\Gamma^{\prime}$ such that $\rho^{\prime}\left(\gamma_{j}\right)=1, j=1, \ldots, n$, i.e. $\rho^{\prime}=\rho \in \operatorname{Hom}\left(\Gamma, \mathbb{C}^{*}\right)$. Consider the diagram

$$
\begin{array}{ccc}
E^{\prime}=\cup \frac{\Omega_{\rho}^{q}\left(\frac{1}{\left.Q_{1}^{\alpha_{1}} \ldots Q_{s}^{\alpha_{s}}, F_{\mu}^{\prime}\right) \cap M_{1}}\right.}{\Omega_{\rho}^{q}\left(1, F_{\mu}^{\prime}\right) \cap M_{1}} & \rightarrow & \cup \frac{\Omega_{\rho}^{q}\left(\frac{1}{Q_{1}^{\alpha_{1}} \ldots Q_{s}^{\alpha_{s}}}, F_{\mu}\right)}{\Omega_{\rho}^{q}\left(1, F_{\mu}\right)}=E \\
\downarrow & \downarrow \\
\widetilde{\mathbb{T}}_{g}^{n} \times \operatorname{Hom}\left(\Gamma, \mathbb{C}^{*}\right) \backslash X & \rightarrow & \mathbb{T}_{g} \times \operatorname{Hom}\left(\Gamma, \mathbb{C}^{*}\right) \backslash X,
\end{array}
$$

where $\widetilde{\mathbb{T}}_{g}^{n}$ is a part of the Teichmüller space $\mathbb{T}_{g, n}[6, \mathrm{p} .81, \mathrm{p} .88]$, the vertical arrows are projections in vector bundles, and the lower horizontal arrow is related to the operation of gluing the punctures, which makes the surface $F \backslash\left\{P_{1}, \ldots, P_{n}\right\}$ into a compact surface $F$ [3]. The upper horizontal arrow will be explained later.

Theorem 3. The diagram above is a commutative diagram of vertical holomorphic vector bundles with isomorphic corresponding fibers and horizontal holomorphic $n !-$ sheeted mappings, where $X=1$ when $q>1$, and $X=L_{g}$ when $q=1$.

Proof. By the Riemann-Roch theorem we find the dimension $i_{\rho, q}\left(\frac{1}{P_{1}^{q_{1}} \ldots P_{n}^{q_{n}}}, F\right)=(2 q-1)(g-$ $-1)+q_{1}+\ldots+q_{n}$. Therefore, $\Omega_{\rho}^{q}\left(1, F^{\prime}\right)$ is an infinite-dimensional vector space.

Now we prove the isomorphism of fibers for fixed $F^{\prime}$ and $F$, where $F$ is obtained from $F^{\prime}$ by glueing up the punctures. For any fixed $\rho \neq 1$ we define the map $\theta$ of a fiber of $E^{\prime}$ over $F^{\prime}$ into 
a fiber of $E$ over $F$, which puts in correspondence to the class $<\omega>=\omega+\Omega_{\rho}^{q}\left(1, F^{\prime}\right) \cap M_{1}$ the class $\langle\omega-\tilde{\omega}\rangle=\omega-\tilde{\omega}+\Omega_{\rho}^{q}(1, F)$ in the following way. If $\omega \in \Omega_{\rho}^{q}\left(\frac{1}{Q_{1}^{\alpha_{1}} \ldots Q_{s}^{\alpha_{s}}}, F^{\prime}\right) \cap M_{1}$, i.e. $(\omega) \geqslant \frac{1}{Q_{1}^{\alpha_{1}} \ldots Q_{s}^{\alpha_{s}}} \cdot \frac{1}{P_{1}^{q_{1}} \ldots P_{n}^{q_{n}}}$, then we put into correspondence $\omega-\tilde{\omega}$, since by Lemma 2 we can choose the differential $\tilde{\omega}$ such that $(\tilde{\omega})=\frac{R_{1} \ldots R_{N}}{P_{1}^{q_{1}} \ldots P_{n}^{q_{n}}}, R_{j} \neq P_{l}, j=1, \ldots, N, l=1, \ldots, n$, and having the same principal parts of Laurent series at all points $P_{j}, j=1, \ldots, n$, as an analytic continuation of the differential $\omega$ to $F$. Then $\omega-\tilde{\omega} \in \Omega_{\rho}^{q}\left(\frac{1}{Q_{1}^{\alpha_{1}} \ldots Q_{s}^{\alpha_{s}}}, F\right)$. Let $\theta\left(\omega+\Omega_{\rho}^{q}\left(1, F^{\prime}\right) \cap\right.$ $\left.M_{1}\right)=\omega-\tilde{\omega}+\Omega_{\rho}^{q}(1, F)$.

We shall show that this mapping is well-defined. Consider another differential $\omega^{\prime}$ from the same equivalency class $<\omega+\Omega_{\rho}^{q}\left(1, F^{\prime}\right) \cap M_{1}>$. It has the same singularities as $\omega$ at all points $Q_{1}, \ldots, Q_{s}$, and its own singularities at the punctures, i.e. $\left(\omega^{\prime}\right) \geqslant \frac{1}{Q_{1}^{\alpha_{1}} \ldots Q_{s}^{\alpha_{s}} P_{1}^{q_{1}^{\prime}} \ldots P_{n}^{q_{n}^{\prime}}}$. Then we choose $\tilde{\omega}^{\prime}$ such that $\omega^{\prime}-\tilde{\omega}^{\prime} \in \Omega_{\rho}^{q}\left(\frac{1}{Q_{1}^{\alpha_{1}} \ldots Q_{s}^{\alpha_{s}}}, F\right)$. Therefore, on the one hand we have $\theta(<\omega>)=<\omega-\tilde{\omega}>$, on the other hand we have $\theta\left(<\omega^{\prime}>\right)=<\omega^{\prime}-\tilde{\omega}^{\prime}>$. Consider the difference of representatives of both classes $\left(\omega-\omega^{\prime}\right)+\left(\tilde{\omega^{\prime}}-\tilde{\omega}\right)=(\omega-\tilde{\omega})-\left(\omega^{\prime}-\tilde{\omega}^{\prime}\right)=\phi \in \Omega_{\rho}^{q}(1, F)$, i.e. $\phi$ is a holomorphic $(\rho, q)$-differential on $F$. Therefore,

$$
\theta(<\omega>)=\omega-\tilde{\omega}+\Omega_{\rho}^{q}(1, F)=\omega^{\prime}-\tilde{\omega}^{\prime}+\phi+\Omega_{\rho}^{q}(1, F)=\omega^{\prime}-\tilde{\omega}^{\prime}+\Omega_{\rho}^{q}(1, F)=\theta\left(<\omega^{\prime}>\right) .
$$

Thus, the map $\theta$ is well defined on the equivalency classes.

Let us establish that $\theta$ is surjective. For any equivalency class $\omega_{0}+\Omega_{\rho}^{q}(1, F)$ we define the class $\omega_{0}+\tilde{\tilde{\omega}}+\Omega_{\rho}^{q}\left(1, F^{\prime}\right)$, where $(\tilde{\tilde{\omega}})=\frac{R_{1} \ldots R_{N}}{P_{1}^{q_{1}} \ldots P_{n}^{q_{n}}}$ on $F$ and $\left(\omega_{0}+\tilde{\tilde{\omega}}\right) \in \Omega_{\rho}^{q}\left(\frac{1}{Q_{1}^{\alpha_{1}} \ldots Q_{s}^{\alpha_{s}}}, F^{\prime}\right) \cap M_{1}$ for some $q_{j} \geqslant 0, j=1, \ldots, n$. Thus, $\theta\left(\omega_{0}+\tilde{\tilde{\omega}}+\Omega_{\rho}^{q}\left(1, F^{\prime}\right) \bigcap M_{1}\right)=\omega_{0}+\Omega_{\rho}^{q}(1, F)$. This can be proved differently. Take a Prym differential with required singularities holomorphic at punctures on $F_{\mu}$ from the bundle in the right hand side of (4) and consider it on the surface with punctures. The map $\theta$ takes it back.

Now let us prove that the mapping of a fixed fiber over $F^{\prime}$ and a fiber over the corresponding surface $F$ is 1 -to-1. Assume that different equivalency classes are mapped by $\theta$ to one class, i.e. $\theta\left(<\omega_{1}>\right)=<\omega_{1}-\tilde{\omega}_{1}>=<\omega_{2}-\tilde{\omega}_{2}>=\theta\left(<\omega_{2}>\right)$, where $\tilde{\omega}_{1} \in \Omega_{\rho}^{q}\left(\frac{1}{P_{1}^{q_{1}} \ldots P_{n}^{q_{n}}}, F\right)$, a $\tilde{\omega}_{2} \in \Omega_{\rho}^{q}\left(\frac{1}{P_{1}^{q_{1}^{\prime}} \ldots P_{n}^{q_{n}^{\prime}}}, F\right)$ and $\left(\omega_{2}-\omega_{1}\right)$ does not belong to $\Omega_{\rho}^{q}\left(1, F^{\prime}\right) \cap M_{1}$. Consider the difference $\left(\omega_{2}-\omega_{1}\right)+\left(\tilde{\omega}_{1}-\tilde{\omega}_{2}\right)=\left(\omega_{2}-\tilde{\omega}_{2}\right)-\left(\omega_{1}-\tilde{\omega}_{1}\right)=\phi \in \Omega_{\rho}^{q}(1, F)$. Therefore $\omega_{2}-\omega_{1}=$ $=\phi+\tilde{\omega}_{2}-\tilde{\omega}_{1} \in \Omega_{\rho}^{q}\left(1, F^{\prime}\right) \cap M_{1}$. This is a contradiction.

Now we prove that $\theta$ is linear. Indeed, for $c_{j} \in \mathbb{C}, j=1,2$, we have equalities

$$
\begin{gathered}
\theta\left[c_{1}\left(\omega_{1}+\Omega_{\rho}^{q}\left(1, F^{\prime}\right) \cap M_{1}\right)+c_{2}\left(\omega_{2}+\Omega_{\rho}^{q}\left(1, F^{\prime}\right) \cap M_{1}\right)\right]= \\
=\theta\left[c_{1} \omega_{1}+c_{2} \omega_{2}+\Omega_{\rho}^{q}\left(1, F^{\prime}\right) \cap M_{1}\right]=c_{1} \omega_{1}+c_{2} \omega_{2}-\left(c_{1} \tilde{\omega}_{1}+c_{2} \tilde{\omega}_{2}\right)+\Omega_{\rho}^{q}(1, F)= \\
=c_{1}\left(\omega_{1}-\tilde{\omega}_{1}\right)+c_{1} \Omega_{\rho}^{q}(1, F)+c_{2}\left(\omega_{2}-\tilde{\omega}_{2}\right)+c_{2} \Omega_{\rho}^{q}(1, F)=c_{1} \theta\left(<\omega_{1}>\right)+c_{2} \theta\left(<\omega_{2}>\right) .
\end{gathered}
$$

Thus, $\theta$ is linear, and we get an isomorphism

$$
\theta: \frac{\Omega_{\rho}^{q}\left(\frac{1}{Q_{1}^{\alpha_{1}} \ldots Q_{s}^{\alpha_{s}}}, F_{\mu}^{\prime}\right) \cap M_{1}}{\Omega_{\rho}^{q}\left(1, F_{\mu}^{\prime}\right) \cap M_{1}} \rightarrow \frac{\Omega_{\rho}^{q}\left(\frac{1}{Q_{1}^{\alpha_{1}} \ldots Q_{s}^{\alpha_{s}}}, F_{\mu}\right)}{\Omega_{\rho}^{q}\left(1, F_{\mu}\right)}
$$


of fibers under these conditions.

Now we lift the set (2) to the set of Prym differentials on $F_{\mu}^{\prime}$, which is obtained from (2) by adding some differential from $\Omega_{\rho}^{q}\left(1, F_{\mu}^{\prime}\right) \cap M_{1}$. All these differentials can be chosen so that they depend holomorphically on $[\mu]$ and $\rho$ on $F_{\mu}^{\prime}$. Thus, we obtain a set of equivalency classes of differentials

$$
\begin{array}{r}
\tau_{\rho, q ; Q_{1}}^{(1)}+\Omega_{\rho}^{q}\left(1, F_{\mu}^{\prime}\right), \tau_{\rho, q ; Q_{1}}^{(2)}+\Omega_{\rho}^{q}\left(1, F_{\mu}^{\prime}\right), \ldots, \tau_{\rho, q ; Q_{1}}^{\left(\alpha_{1}\right)}+\Omega_{\rho}^{q}\left(1, F_{\mu}^{\prime}\right), \tau_{\rho, q ; Q_{2}}^{(1)}+\Omega_{\rho}^{q}\left(1, F_{\mu}^{\prime}\right), \ldots \\
\ldots, \tau_{\rho, q ; Q_{2}}^{\left(\alpha_{2}\right)}+\Omega_{\rho}^{q}\left(1, F^{\prime}\right), \ldots, \tau_{\rho, q ; Q_{s}}^{(1)}+\Omega_{\rho}^{q}\left(1, F_{\mu}^{\prime}\right), \ldots, \tau_{\rho, q ; Q_{s}}^{\left(\alpha_{s}\right)}+\Omega_{\rho}^{q}\left(1, F_{\mu}^{\prime}\right)
\end{array}
$$

which correspond to Prym differentials from $(2)$, on $F_{\mu}^{\prime}$. It is a basis of locally holomorphic sections of the vector bundle $E^{\prime}$. Consequently, both these bundles $E$ and $E^{\prime}$ are holomorphic vector bundles of rank $d$ over mentioned bases.

The operation of gluing up the punctures that makes $F^{\prime}$ into $F$ defines an $n$ !-sheeted holomorphic mapping from $\widetilde{\mathbb{T}}_{g}^{n}$ onto $\mathbb{T}_{g}$. Here, over each surface $F$ with fixed points $P_{1}, \ldots, P_{n}$ there are $n$ ! surfaces $F^{\prime}$.

Thus, we have proved commutativity of the diagram (4) with required properties.

\section{Spaces of univalent differentials}

Lemma 3. For each divisor $P_{1}^{q_{1}} \cdot \ldots \cdot P_{n}^{q_{n}}, q_{j} \geqslant 0, j=1, \ldots, n$, and $q>1$ on $F_{\mu}$ of genus $g \geqslant 2$ there exists a differential $\tilde{\omega} \in \Omega^{q}\left(\frac{1}{P_{1}^{q_{1}} \cdot \ldots \cdot P_{n}^{q_{n}}}, F_{\mu}\right)$ with the divisor $(\tilde{\omega})=\frac{R_{1}, \ldots, R_{N}}{P_{1}^{q_{1}} \cdot \ldots \cdot P_{n}^{q_{n}}}$, where $R_{j} \neq P_{l}, l=1, \ldots, n, j=1, \ldots, N, N=(2 g-2) q+q_{1}+\ldots+q_{n}$, and any given principal parts of Laurent series at $P_{j}, j=1, \ldots, n$. This differential depends locally holomorphically on moduli $[\mu]$ of the surface $F_{\mu}$.

The proof is analogous to the proof of Lemma 2.

Denote by $\Omega^{q}\left(\frac{1}{Q_{1}^{\alpha_{1}} \ldots Q_{l}^{\alpha_{l}} Q_{l+1} \ldots Q_{s}} ; F_{\mu}\right)$ for $q>1$ the space of $q$-differentials on $F_{\mu}$ that are multiple of the divisor $\frac{1}{Q_{1}^{\alpha_{1}} \ldots Q_{l}^{\alpha_{l}} Q_{l+1} \ldots Q_{s}}$, where $\alpha_{1}, \ldots, \alpha_{l} \geqslant 2, s \geqslant 1,0 \leqslant l \leqslant s$ and the points $Q_{1}, \ldots, Q_{s}$ are distinct, and by $\Omega^{q}\left(1 ; F_{\mu}\right)$ denote the subspace of holomorphic $q$-differentials on $F_{\mu}$.

By the Riemann-Roch theorem for $q$-differentials we find the dimensions of these spaces. It is known that $\operatorname{dim} \Omega^{q}\left(1 ; F_{\mu}\right)=(2 q-1)(g-1)$ for $q>1$. Moreover,

$$
\begin{aligned}
& i_{q}\left(\frac{1}{Q_{1}^{\alpha_{1}} \ldots Q_{l}^{\alpha_{l}} Q_{l+1} \ldots Q_{s}}\right)=(g-1)(2 q-1)-\operatorname{deg}\left(\frac{1}{Q_{1}^{\alpha_{1}} \ldots Q_{l}^{\alpha_{l}} Q_{l+1} \ldots Q_{s}}\right)+ \\
& +r\left(Z^{q-1} Q_{1}^{\alpha_{1}} \ldots Q_{l}^{\alpha_{l}} Q_{l+1} \ldots Q_{s}\right)=(g-1)(2 q-1)+\alpha_{1}+\ldots+\alpha_{l}+s-l(\geqslant 4) .
\end{aligned}
$$

Therefore, $\operatorname{dim} \Omega^{q}\left(\frac{1}{Q_{1}^{\alpha_{1}} \ldots Q_{l}^{\alpha_{l}} Q_{l+1} \ldots Q_{s}} ; F_{\mu}\right) / \Omega^{q}\left(1 ; F_{\mu}\right)=\alpha_{1}+\ldots+\alpha_{l}+s-l(\geqslant 1)$.

Consider the sets of $q$-differentials:

$$
\tau_{q ; Q_{1}}^{(1)}, \tau_{q ; Q_{1}}^{(2)}, \ldots, \tau_{q ; Q_{1}}^{\left(\alpha_{1}\right)}, \ldots, \tau_{q ; Q_{l}}^{(1)}, \tau_{q ; Q_{l}}^{(2)}, \ldots, \tau_{q ; Q_{l}}^{\left(\alpha_{l}\right)}, \tau_{q ; Q_{1} Q_{l+1}}, \ldots, \tau_{q ; Q_{1} Q_{s}}
$$

for $l \geqslant 1, q>1$;

$$
\tau_{q ; Q_{1}}^{(1)}, \tau_{q ; Q_{1} Q_{2}}, \ldots, \tau_{q ; Q_{1} Q_{s}}
$$

for $l=0, q>1$ on $F_{\mu}$. 
Proposition 2 ([6]). The bundle

$$
\cup \Omega^{q}\left(\frac{1}{Q_{1}^{\alpha_{1}} \ldots Q_{l}^{\alpha_{l}} Q_{l+1} \ldots Q_{s}} ; F_{\mu}\right) / \Omega^{q}\left(1 ; F_{\mu}\right)
$$

is a holomorphic vector bundle of rank $\alpha_{1}+\ldots+\alpha_{l}+s-l$ over $\mathbb{T}_{g}$, where $g \geqslant 2, \alpha_{1}, \ldots, \alpha_{l} \geqslant 2$, $s \geqslant 1,0 \leqslant l \leqslant s, q>1$ and the points $Q_{1}, \ldots, Q_{s}$ are distinct. The equivalency classes of q-differentials from (5), (6) form a base of locally holomorphic sections of this bundle over $\mathbb{T}_{g}$.

Consider the diagram

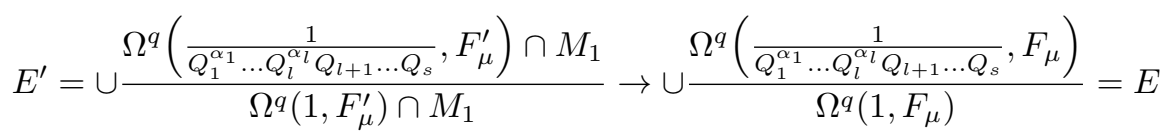

$$
\begin{aligned}
& \begin{aligned}
\downarrow & \downarrow \\
\widetilde{\mathbb{T}}_{g}^{n} & \rightarrow \quad \mathbb{T}_{g} .
\end{aligned}
\end{aligned}
$$

Теорема 4. The diagram (7) is commutative; vertical arrows stand for holomorphic vector bundles with isomorphic corresponding fibers, horisontal arrows are for holomorphic n!-sheeted mappings over bases from $\widetilde{\mathbb{T}}_{g}^{n}$ (a part of the Teichmüller spaces $\mathbb{T}_{g, n}$ ) and a Teichmüller space $\mathbb{T}_{g}$.

The proof follows the proof of Theorem 3 together with Lemma 3 and Proposition 2.

The work was financially supported by RFBR (grants no. 15-01-07906, no. 18-01-00420) and the grant of the Russian Federation Government for scientific research under the supervision of leading scientist at Siberian Federal University (contract no. 14. Y26.31.0006).

\section{References}

[1] A.B.Bogatyrev, Real meromorphic differentials: a language for describing meron configurations in planar magnetic nanoelements, Theoretical and Mathematical Physics, 193(2017), no. $1,1547-1559$.

[2] L.Ahlfors, L.Bers, The spaces of Riemann surfaces and quasi-conformal mappings, IL, Moscow, 1961 (in Russian).

[3] H.M.Farkas, I.Kra, Riemann surfaces, Springer, New-York, Grad. Text's Math. V. 71, 1992.

[4] C.J.Earle, Families of Riemann surfaces and Jacobi varieties, Annals of Mathematics, 107(1978), 255-286.

[5] V.V.Chueshev, Multiplicative functions and Prym differentials on a variable compact Riemann surface, Part 2, KemGU, Kemerovo, 2003 (in Russian).

[6] V.V.Chueshev, M.I.Tulina, Prym differentials on a variable compact Riemann surface, Matematicheskie Zametki, 95(2014) no. 3, 457-474 (in Russian).

[7] A.S.Mischenko, Vector bundles and their applications, Nauka, Moscow, 1984 (in Russian).

[8] A.V.Chueshev, V. V.Chueshev, The residue theorem and an analog of P. Appell's formula for finite Riemann surface, Science Evolution, 1(2016), no. 1, 40-45. 


\section{Векторное расслоение дифференциалов Прима над пространствами Тейхмюллера поверхностей с проколами}

Александр В. Чуешев

Виктор В. Чуешев

Институт фундаментальных наук Кемеровский государственный университет Красная, 6, Кемерово, 650043

Россия

В работе исследуются мультипликативные мероморфные функции и дифференциаль на римановых поверхностях конечного типа. Доказан аналог формулы П. Аппеля о разложении мультипликативной функции с полюсами любых кратностей в сумму элементарных интегралов Прима. Построены явные базисы для ряда важсных фактор-пространств. Доказана теорема о послойном изоморфизме векторных расслоений и n!-листных отображений над пространствами Тейхмюллера. Эта теорема дает важную связв между пространствами дифференциалов Прима (абелевых дифференциалов) на компактной римановой поверхности и на римановой поверхности конечного muna.

Ключевые слова: пространства Тейхмюллера римановых поверхностей конечного типа, дифференциалы Прима, векторные расслоения, группа характеров, многообразия Якоби. 